ISSN 1112-9867

Available online at

http://www.jfas.info

\title{
CHARACTERIZATION QUALITATIVE OF SOEL FOR A VALORIZATION BEST OF THE BIOMASS PRODUCED
}

\author{
Y. M'Sadak* and I. Saad \\ Département du Génie des Systèmes Horticoles et du Milieu Naturel \\ Institut Supérieur Agronomique de Chott-Mariem, Université de Sousse, Tunisie
}

Received: 03 July 2014 / Accepted: 17 December 2014 / Published online: 15 January 2015

\begin{abstract}
The main objective of this study was to qualitatively characterize biomass of Solanum elaeagnifolium Cav. (or SOEL) to highlight the essential alternatives its valorization. At first, we studied the potential of composting biomass of this plant. The physico-chemical characterization of biomass silverleaf nightshade has detected a wealth in organic carbon, which highlighted the potential value of this plant in the Co-composting with other biodegradable waste. In a second step, we assessed the possibility to develop this plant in the area of treatment in textile effluents by biosorption of dyes on fibers from the biomass of this plant, especially after the discovery of high levels of cellulose in its different organs.
\end{abstract}

Keywords: Biomass of SOEL; Physico-chemical characterization; Composting; Characterization cellulosic; Biosorption.

\section{INTRODUCTION}

La morelle jaune (solanum elaeagnifolium Cav.), dénommée simplement SOEL, est une mauvaise herbe vivace rhizomateuse et drageonnante. Originaire du Continent Américain, elle est naturalisée dans plusieurs pays du monde, tout en constituant une menace sérieuse pour la biodiversité. Présentement, elle est considérée une plante exotique envahissante (PEE) dans les cinq continents [1].

Author Correspondence, e-mail: msadak.youssef@yahoo.fr

Tel.: +21673 327 546; fax: +21673327591.

ICID: 1131851 
Une fois établie, son contrôle devient très difficile. Son système souterrain, très développé, est constitué d'une racine principale verticale pénétrant jusqu'à $2 \mathrm{~m}$ de profondeur et de racines latérales atteignant $2 \mathrm{~m}$ de longueur et possédant des bourgeons donnant naissance à des rhizomes [2].

SOEL fleurit du printemps jusqu'à la fin de l'été. Elle fructifie de l'été à l'automne. Cette plante, présentant une grande variabilité morphologique, peut mesurer jusqu'à $1 \mathrm{~m}$ de haut. Ses fruits, intermédiaires entre baie et capsule, sont initialement sphériques, verts devenant jaune-orange à maturité et mesurent entre 10 et $15 \mathrm{~mm}$ de diamètre. A maturité les fruits sont secs. Chaque fruit contient entre 60 et 120 graines recouvertes d'une substance mucilagineuse. Les graines de 2-3 mm ressemblent aux graines de tomates. Un pied peut produire jusqu'à 200 baies par saison [3].

L'envahissement incontrôlé des terres agricoles par les PEE est une problématique d'actualité, particulièrement, dans les pays du bassin méditerranéen. En Tunisie, SOEL a été signalée pour la première fois en 1985, dans la délégation de Sbikha du gouvernorat de Kairouan, véritable carrefour qui relie plusieurs régions du pays [4]. En 2004, elle est devenue une mauvaise herbe très redoutable dans cette délégation où elle est abondante et fréquente au niveau des cultures irriguées ainsi que le long des bordures des routes et les bords des rivières. Dans les délégations de Chébika, Kairouan Sud et Kairouan Nord, elle n'est que localement abondante. Dans le reste du gouvernorat, elle est peu fréquente [5]. Cette plante n'est plus confinée au seul gouvernorat de Kairouan et elle a été signalée dans d'autres gouvernorats ; Sousse, Sidi Bouzid, Mahdia, Sfax et Zaghouan. En 2005, la superficie totale envahie par cette plante a été estimée à 20000 ha [6].

La dissémination des graines de SOEL par divers agents (fumier ovin, matériel agricole, plants en mottes, paille, vent, eau d'irrigation) a élargi le secteur de propagation de la plante [7]. C'est l'une des PEE les plus dangereuses, à cause des dégâts néfastes qu'elle répercute sur l'environnement, l'économie et sur les récoltes agricoles, du fait qu'elle est capable d'envahir de grandes superficies agricoles en une durée de temps très courte, ce qui rend la lutte contre cette PEE très difficile. Par conséquent, l'élimination de l'impact négatif provoqué par cette espèce envahissante dans les régions méditerranéennes est devenue indispensable. En absence d'un cadre règlementaire et d'une stratégie nationale de gestion adaptative, les PEE, en particulier SOEL, continuent à menacer l'environnement et l'agriculture. Leur maîtrise exige la coopération de toutes les parties concernées pour définir et mettre en œuvre un plan d'action pluriannuel de gestion de ces envahisseurs [8]. 
Afin de pallier cette situation dans le cadre d'une gestion rationnelle et durable, sans pour autant tendre vers une extermination systématique de cette plante, à cause de ses caractéristiques biochimiques intéressantes identifiées, deux solutions essentielles ont été proposées dans le présent travail. Il s'agit de caractériser qualitativement la biomasse de SOEL et d'étudier les possibilités de sa valorisation, d'une part, par compostage (en se basant sur sa qualité physico-chimique), et d'autre part, comme biosorbant des colorants rejetés dans les eaux usées de l'industrie textile (en se référant à une caractérisation cellulosique).

\section{MATÉRIEL ET MÉTHODES}

\section{Évaluation physico-chimique de la biomasse partielle}

\section{Considérations générales}

Les analyses ont été réalisées pour deux stades de croissance de morelle jaune. La première collecte des plantes était réalisée au 18/01/2010. La deuxième collecte était réalisée au 10/04/2010. Les stades de croissance de la plante à ces dates ont été identifiés respectivement au stade Grenaison avancé (Graines mûres) et au stade bouton floral avancé (Boutons floraux prêts à ouvrir avec extrémité colorée). Les plantes de SOEL (Photo 1) ont été arrachées manuellement à partir des bordures de parcelles cultivées de la région de Chott-Mariem (Sousse, Tunisie).

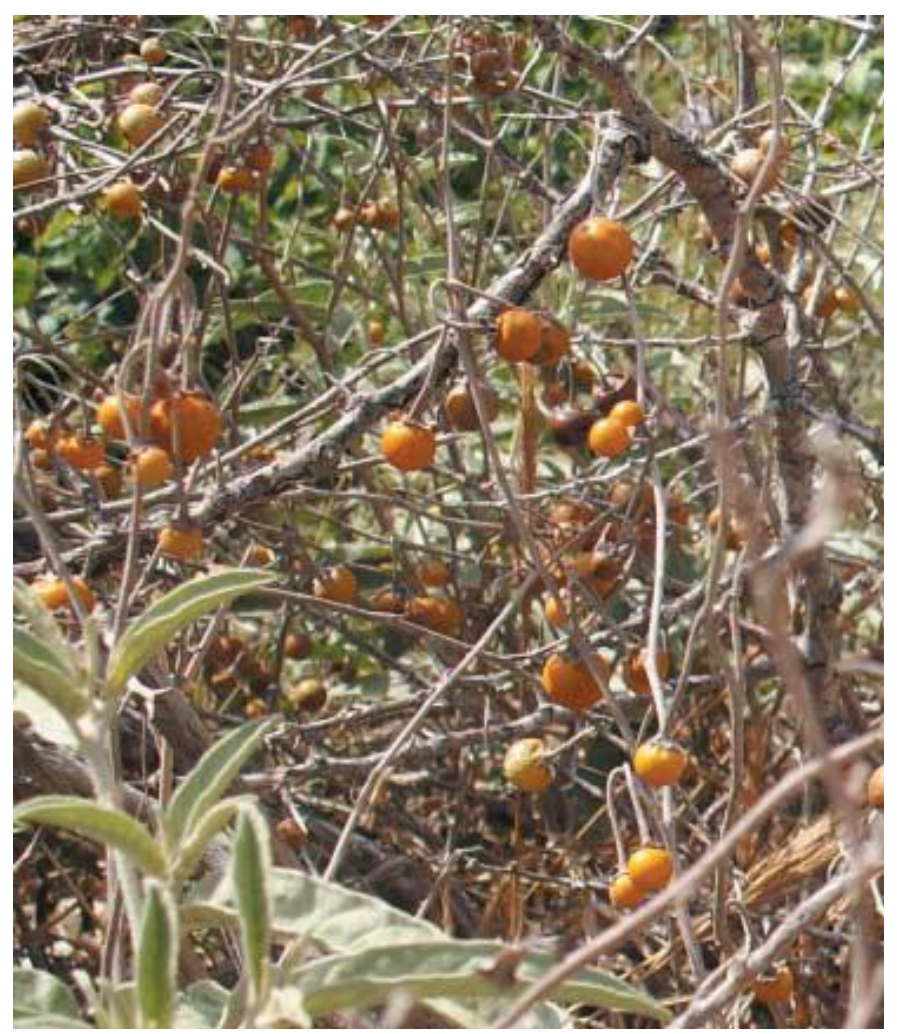

Photo 1 : Morelle jaune (Solanum elaeagnifolium). Cliché (Sarah Brunel, 2006) [9] 


\section{Détermination de la teneur en matière sèche initiale et en humidité}

\section{Matériel}

Plantes de morelle jaune, étuve, balance, cages métalliques.

\section{Méthode}

Juste après l'arrachage manuel relatif à chaque stade de croissance, les plantes arrachées ont été scindées en trois parties (racines, tiges et feuilles + baies pour la première récolte ou feuilles + boutons floraux pour la deuxième récolte) servant comme échantillons pour les analyses effectuées. Chaque échantillon a été mis dans une cage métallique et a été pesé pour déterminer son poids frais (Pf). Les échantillons étaient ensuite introduits dans l'étuve durant 48 heures à $60^{\circ} \mathrm{C}[10]$.

Après séchage à l'étuve, les échantillons ont été pesés de nouveau pour déterminer leur poids $\sec (\mathbf{P s})$.

La teneur en Matière Sèche initiale (MSi) de chaque échantillon a été calculée selon la formule ci-après.

$$
\% \mathbf{M S i}=(\mathbf{P s} / \mathbf{P f}) \times 100
$$

La teneur en eau ou Humidité $(\mathrm{H})$ des échantillons a été calculée comme suit.

$$
\% \mathbf{H}=((\mathbf{P f}-\mathbf{P s}) / \mathrm{Pf}) \times 100
$$

\section{Détermination de la teneur en matière sèche analytique}

\section{Matériel}

Plantes de morelle jaune, broyeur, étuve, sachets plastiques, creusets en porcelaine, dessiccateur.

\section{Méthode}

Les échantillons, après séchage à l'étuve et détermination de la teneur en matière sèche initiale, étaient broyés à l'aide d'un broyeur. Après broyage, les échantillons étaient conservés dans des sachets plastiques bien fermés et étiquetés.

Selon AOAC [11], un échantillon séché dans une étuve à température inférieure à $105^{\circ} \mathrm{C}$ ne peut jamais être dépourvu totalement d'eau. Cette teneur en eau qui est généralement de $( \pm 5 \%)$, peut être affectée dans le temps par l'humidité de l'air ambiant (particulièrement au moment du stockage). La connaissance de la teneur en cette petite quantité d'eau permet d'exprimer les résultats des différentes analyses à réaliser sur l'échantillon en pourcentage de la matière sèche. Ceci consiste en fait à corriger les prises d'essai utilisées au moment des 
analyses par rapport à leur quantité de matière sèche réelle (teneur en matière sèche analytique). Ce dosage doit être absolument réalisé au moment des analyses pour éviter les erreurs dues aux changements de cette teneur dans le temps.

Le principe AOAC [11] consiste à éliminer cette fine quantité d'eau par séchage à l'étuve à $105^{\circ} \mathrm{C}$ durant 24 heures. Des creusets en porcelaine préalablement identifiés et pesés à vide (Pt) étaient remplis chacun par $3 \mathrm{~g}$ de chaque échantillon et étaient pesés de nouveau pour déterminer le poids des creusets pleins P1. Les creusets étaient ensuite mis dans l'étuve à $105^{\circ} \mathrm{C}$ pendant 24 heures. Après séchage et refroidissement dans un dessiccateur, les creusets pleins étaient de nouveau pesés pour déterminer $\mathrm{P} 2$.

La teneur en Matière Sèche analytique (MSa) a été déterminée selon la formule ci-après.

$$
\% \mathrm{MSa}=((\mathbf{P} 2-\mathbf{P t}) /(\mathbf{P 1}-\mathbf{P t})) * 100
$$

\section{Détermination de la teneur en matière minérale}

\section{Matériel}

Echantillons broyés de morelle jaune, four à moufle, creusets en porcelaine, balance de précision, dessiccateur

\section{Méthode}

Les échantillons séchés à $105^{\circ} \mathrm{C}$ étaient ensuite calcinés pour déterminer la teneur en matière minérale. Les creusets étaient alors mis dans un four à moufle de type à $550^{\circ} \mathrm{C}$ pendant 6 heures [11]. Après refroidissement dans le dessiccateur, les échantillons étaient de nouveau pesés pour déterminer leurs poids après calcination $\mathrm{P} 3$.

La teneur en Matière minérale (MM) a été calculée selon la formule ci-après.

$$
\% \mathbf{M M}=((\mathbf{P 3}-\mathbf{P t}) /(\mathbf{P 2}-\mathbf{P t})) * 100
$$

\section{Teneur en matière organique totale et carbone organique total}

La teneur en matière organique totale (MOT) est déterminée à partir de la teneur en matière minérale (MM).

$$
\% \text { MOT }=100-\% \text { MM }
$$

Le taux de carbone organique total (COT) est déduit de la teneur en matière organique totale (MOT) selon la formule de Golueke [12] ci-après.

$$
\% \mathrm{COT}=\% \text { MOT } / 1,8
$$




\section{Teneur en azote total}

\section{Matériel}

Echantillons broyés de morelle jaune, digesteur, distillateur, erlenmeyers, burette graduée, agitateur, eau distillée, matras à digestion, acide sulfurique concentré $\left(\mathrm{H}_{2} \mathrm{SO}_{4}, \mathrm{~d}=1,84\right)$, acide chlorhydrique dilué $(\mathrm{HCl}, \mathrm{N} / 10)$, Soude $(\mathrm{NaOH})$, catalyseur, acide borique dilué $\left(\mathrm{H}_{3} \mathrm{BO}_{3}\right.$ dilué à 4\%).

\section{Méthode}

Le taux d'azote est déterminé par la méthode de Kjeldhal. L'échantillon subit une digestion ou minéralisation par l'acide sulfurique concentré en présence d'un catalyseur. L'azote organique se transforme en azote ammoniacal (sulfate d'ammonium $\left(\mathrm{NH}_{4}\right)_{2} \mathrm{SO}_{4}$ ) qui est déplacé par la soude et reçu dans une solution titrée d'acide borique. La quantité d'ammoniac (NH3) présente est mesurée par titration avec l'acide chlorhydrique.

$$
\begin{gathered}
\% \mathrm{NT}=((\mathrm{Ve}-\mathrm{Vb}) * 0,14) / \mathrm{Pe} \\
\% \mathrm{MAT}=\% \mathrm{NT} * 6,25
\end{gathered}
$$

Avec :

NT : teneur d'azote total en $\%$ de matière sèche.

Ve : volume de $\mathrm{HCl}$ ajouté (ml).

Vb : volume de $\mathrm{HCl}$ ajouté pour l'essai à blanc $(\mathrm{ml})$.

Pe : Prise d'essai en g.

MAT : matière azotée totale en $\%$ de matière sèche.

\section{Détermination du rapport $\mathrm{C} / \mathrm{N}$}

Le rapport Carbone/Azote $(\mathrm{C} / \mathrm{N})$ est déterminé par :

\section{$\mathrm{C} / \mathrm{N}=\mathrm{COT} / \mathrm{NT}$}

\section{Potentiel Hydrogène}

\section{Matériel}

Des erlenmeyers, eau distillée, broyat de chaque partie de la plante, $\mathrm{pH}$ mètre électronique et agitateur électronique.

\section{Méthode}

On a mis $4 \mathrm{~g}$ du broyat de chaque échantillon dans un erlenmeyer contenant $100 \mathrm{ml}$ d'eau distillée et on a fait une agitation pendant une 1/2 heure. On a laissé la solution reposer pendant 2 heures pour que les sels soient totalement dissous dans l'eau. Ensuite, on a mesuré le 
potentiel Hydrogène $(\mathrm{pH})$ de chaque solution à l'aide d'un $\mathrm{pH}$ mètre électronique après étalonnage de l'appareil. Les mesures ont été accomplies en triplicata.

\section{Conductivité électrique et salinité}

\section{Matériel}

Des erlenmeyers, eau distillée, broyat de chaque partie de la plante, conductimètre électronique et agitateur électronique.

\section{Méthode}

La conductivité électrique (CE) est la conductance d'une colonne d'eau comprise entre deux électrodes métalliques de $1 \mathrm{~cm}^{2}$ de surface et séparées l'une de l'autre de $1 \mathrm{~cm}$. Elle est mesurée par le conductimètre en $(\mu \mathrm{S} / \mathrm{cm})$, qui donne aussi une idée sur la quantité de sels et d'ions existantes dans la solution (salinité). Les mesures ont été réalisées pour les mêmes solutions préparées pour mesurer le $\mathrm{pH}$ de chaque partie de la plante. La salinité représente le taux des sels totaux dans le substrat. Elle est déterminée en se basant sur la formule de conversion ci-après.

$$
1 \mathrm{mS}=0,7 \mathrm{~g} / \mathrm{l}
$$

\section{Évaluation cellulosique de la biomasse partielle}

\section{Matériel}

Broyat de chaque partie de la plante, Fibertec, balance de précision, creusets en porcelaine, solution d'acide sulfurique $\mathrm{H}_{2} \mathrm{SO}_{4}(0,128 \mathrm{M})$, solution d'hydroxyde de potassium $\mathrm{KOH}$ $(0,223 \mathrm{M})$, étuve, four à moufle.

\section{Méthode}

La méthode de Weende $[11,13]$ a été adoptée pour la détermination du contenu en fibres ou cellulose brute (CB) dans les différentes parties de la plante étudiée. Les creusets étaient numérotés et pesés à vide $(\mathrm{Pt})$.Une prise de $1 \mathrm{~g}$ de chaque échantillon broyé a été réalisée à l'aide d'une balance de précision (P1). Chaque échantillon a subi une hydrolyse acide par $150 \mathrm{ml}$ d'acide sulfurique $\mathrm{H}_{2} \mathrm{SO}_{4}(0,128 \mathrm{M})$ et une autre alcaline par $150 \mathrm{ml}$ d'hydroxyde de potassium $\mathrm{KOH}(0,223 \mathrm{M})$. Les deux hydrolyses étaient séparées par une filtration et un rinçage à l'eau chaude. Cette opération était réalisée à l'aide d'un appareil dit « Fibertec 2010, Hot Extractor ». L'échantillon était ensuite séché à l'étuve à une température de $100^{\circ} \mathrm{C}$ pendant 6 heures, puis il a été pesé (P2). Enfin, il a été mis au four à $500^{\circ} \mathrm{C}$ durant 3 heures ensuite, il a été de nouveau pesé (P3). Le résidu sec constitue la cellulose brute qui comprend la cellulose vraie, l'hémicellulose et la lignine. Les mesures étaient accomplies en duplicata.

$$
\% \mathrm{CB}=((\mathbf{P 2}-\mathbf{P 3}) /(\mathbf{P 1}-\mathbf{P t})) * 100
$$


La teneur en Cellulose Brute en \% de matière sèche est déterminée par rapport à la teneur en Matière Sèche analytique de l'échantillon MSa.

$$
\mathrm{CB}(\% \mathrm{MS})=(\% \mathrm{CB}) /(\% \mathrm{MSa})
$$

\section{RÉSULTATS ET DISCUSSION}

\section{Considérations générales}

L'évaluation qualitative de la biomasse de la plante, tant entière que partielle, est quasiabsente dans la littérature consultée. En Tunisie, les travaux de recherche à ce sujet sont rares : la seule étude disponible, se rapporte à une caractérisation physico-chimique et cellulosique, élaborée par Trimech [14]. Les teneurs en matière sèche initiale, matière sèche analytique, matière minérale, matière organique, matière azotée totale et cellulose brute ont été déterminées uniquement pour la plante entière pour trois stades de croissance de SOEL, à savoir: Début végétation, Bouton floral avancé et Floraison avancée. Une telle étude intéressante, a été approfondie en ayant recours à une détermination de paramètres physicochimiques et cellulosiques touchant notamment la biomasse partielle relative aux trois parties de la plante pour deux stades de croissance.

\section{Caractérisation physico-chimique de la biomasse partielle de SOEL}

Les résultats des analyses relatifs à la caractérisation physico-chimique des différentes biomasses de morelle jaune et selon les deux stades de croissance étudiés sont donnés dans le tableau 1 ci-après. Tenant compte de l'absence des normes tunisiennes pour le compostage des déchets, la discussion des résultats fera appel à la norme standard française NF U 44-051 [15].

A priori, la morelle jaune est un déchet sec et riche en carbone, donc difficilement fermentescible. Cependant, la nature exacte de cette plante varie avec la période d'arrachage et l'organe de la plante à considérer.

Une collecte précoce des plantes de SOEL (Stade bouton floral) aura par conséquence des fortes teneurs en eau et en azote, particulièrement au niveau de la frondaison (Teneurs en azote total et en eau respectivement de 3,6\% et de 69,6\% au niveau de la partie feuilles avec boutons floraux), en partie due à la présence des grandes feuilles vertes de la plante. 
Tableau 1. Caractérisation physico-chimique de la biomasse selon deux stades de croissance

\begin{tabular}{|c|c|c|c|c|c|c|c|}
\hline & \multicolumn{3}{|c|}{ Stade bouton floral avancé } & \multicolumn{3}{|c|}{ Stade grenaison avancé } & \multirow{2}{*}{$\begin{array}{l}\text { Norme } \\
\text { Standard }\end{array}$} \\
\hline & Racines & Tiges & Feuilles+Boutons & Racines & Tiges & Feuilles+Baies & \\
\hline $\operatorname{MSi}(\%)$ & 54,2 & 35,7 & 30,4 & 47,6 & 43,9 & 76,3 & \\
\hline $\mathrm{H}(\%)$ & 45,8 & 64,3 & 69,6 & 52,4 & 56,1 & 23,7 & $<60$ \\
\hline $\operatorname{MSa}(\%)$ & 96,0 & 97,1 & 95,0 & 97,0 & 97,1 & 96,7 & \\
\hline MM (\%) & 8,5 & 5,7 & 12,0 & 8,2 & 5,7 & 8,3 & \\
\hline MOT (\%) & 91,5 & 94,3 & 88,0 & 91,8 & 94,3 & 91,7 & \\
\hline COT (\%) & 50,9 & 52,4 & 48,9 & 51,0 & 52,4 & 50,9 & \\
\hline MAT $(\%)$ & 9,5 & 4,9 & 22,0 & 8,1 & 4,4 & 13,8 & \\
\hline NT (\%) & 1,5 & 0,8 & 3,6 & 1,3 & 0,7 & 2,2 & {$[0,7-2]$} \\
\hline $\mathrm{C} / \mathrm{N}$ & 33,5 & 67,1 & 13,7 & 39,5 & 73,7 & 23,1 & {$[30-35]$} \\
\hline $\mathrm{pH}$ & 6,0 & 5,5 & 5,9 & 6,0 & 5,9 & 5,3 & {$[6-8]$} \\
\hline $\mathrm{CE}(\mathrm{mS} / \mathrm{cm})$ & 2,4 & 2,6 & 5,0 & 2,3 & 3,3 & 4,0 & \\
\hline Salinité (g / 1) & 1,7 & 1,8 & 3,5 & 1,6 & 2,3 & 2,8 & \\
\hline
\end{tabular}

Le compostage de cette partie de la plante toute seule provoquerait une perte d'azote s'accompagnant de nuisances olfactives ( $\mathrm{C} / \mathrm{N}$ trop bas en comparaison avec les normes standards). Par contre, cette partie est intéressante en mélange avec les autres parties de la plante (compostage de la biomasse totale de SOEL), car elle fournit les conditions optimales pour la fermentation permettant l'amorçage du compostage sans arrosage préalable d'un produit assez sec comme les tiges. Donc, le compostage de la plante entière (différentes parties de la plante ensemble) à ce stade de croissance semble être plus faisable, puisque l'excès d'azote de la partie aérienne de SOEL serait comblé par l'excès de teneurs en carbone au niveau des racines $(\mathrm{C} / \mathrm{N}=33,5 \%)$ et surtout au niveau des tiges $(\mathrm{C} / \mathrm{N}=67,1 \%)$. Ces deux parties de la plante peuvent être considérées comme agent structurant permettant à la fois la fourniture du carbone organique aux microorganismes dont la consommation par ces derniers est 15 à 30 fois plus importante que celle d'azote [16] et l'oxygène nécessaire à leur survie [17].

Une collecte tardive des plantes de SOEL (Stade grenaison avancé) aura par conséquence une diminution des teneurs en eau et en azote (Teneurs en azote total et en eau respectivement de 2,2\% et de 23,7\% au niveau des feuilles avec les baies), en partie due à l'élaboration de tissus de soutien fortement lignifiés (Teneurs en carbone trop élevées). Le compostage de la biomasse partielle ou même totale de SOEL semble être non faisable pour ce stade de 
croissance, par contre, le Co-compostage de la plante avec un produit fermentescible (tontes de gazon fraîches, fumiers, lisiers, fientes, ...) semble être plus logique, à condition de bien maîtriser le ratio de mélange à appliquer. Les tiges et les racines de la plante, déchets ligneux peu fermentescibles, peuvent être stockées (même plusieurs mois) pour les utiliser comme structurants en mélange avec les déchets plus fermentescibles difficiles à stocker (pouvant être la même plante mais à un stade précoce).

De point de vue $\mathrm{pH}$, le bon développement des bactéries et des champignons responsables de la dégradation de la matière organique est assuré par des valeurs de $\mathrm{pH}$ respectivement proches de la neutralité (6 à 8) et légèrement acides $(5,5$ à 8,0) [12]. D'après le tableau 1, on constate que pour les deux stades de croissance étudiés, le pH des différentes parties de la plante est proche ou même dans les normes (Tableau 1), donc favorable à la vie et à l'activité microbienne responsable de la dégradation de la matière organique pendant le processus de compostage à mettre en œuvre.

Concernant la $\mathrm{CE}$, on peut dire qu'elle est acceptable pour tous les échantillons étudiés sauf pour la partie feuilles et boutons floraux, relative au stade bouton floral avancé, où l'on observe une conductivité électrique de $\mathrm{CE}=5 \mathrm{mS} / \mathrm{cm}$. Cette $\mathrm{CE}$ assez élevée, due en fait à la physiologie de la plante (accumulation des sels au niveau des boutons floraux à ce stade de croissance), pourrait être réduite par lessivage suite aux arrosages qui seront pratiqués au cours du processus de compostage.

La caractérisation physico-chimique de la morelle jaune a permis de décider quant à l'aptitude de la plante à être compostée. Il s'est avéré que cette dernière présente des critères assurant la possibilité de compostage de sa biomasse totale à un stade de croissance précoce et de Co-compostage avec un produit fermentescible pour un stade de croissance adulte. Mais, le compostage ou Co-compostage de cette plante ne doit pas être un facteur de sa dissémination. Le compost produit ne doit présenter aucun risque de germination ou de repousses de morelle jaune. Devant une telle situation, une tentative de contrôle de la propagation de cette plante par les semences (graines et baies) a été entreprise. Pour cette raison, M'Sadak et al. [18] ont mené une étude préliminaire pour évaluer l'effet des hautes températures sur la viabilité des graines de morelle jaune. Les semences de morelle jaune ont été introduites dans l'étuve et ont été traitées à 40,60 et $80^{\circ} \mathrm{C}$ durant 1,2 et 3 jours pour chaque température. Ces traitements ont été effectués, d'une part, sur les graines issues des baies de morelle jaune, et d'autre part, sur les baies de la plante, afin de tester, entre autres, l'effet de la paroi des baies sur la capacité germinative des graines. L'effet température ainsi que l'effet temps 
d'exposition ont montré des différences statistiquement significatives, alors que l'effet nature des semences s'est dévoilé non significatif, et par conséquent, l'enveloppe extérieure des baies n'a pas manifesté un rôle protecteur de la capacité germinative des graines de SOEL. Il a été relevé que, quelque soit la nature des semences (graines traitées ou graines issues des baies traitées), le taux de germination nul a été acquis pour la température de $60^{\circ} \mathrm{C}$ et pour un temps d'exposition de 3 jours, voire 2 jours. La capacité germinative des semences traitées à $80^{\circ} \mathrm{C}$ était nulle dès le premier jour d'exposition, alors que celle des semences traitées à $40^{\circ} \mathrm{C}$ est voisine de celle des semences non traitées (témoin) pour les trois temps d'exposition en étuve, voire même plus élevée. Un tel résultat pourrait expliquer la forte prolifération de cette plante envahissante, déjà adaptée à des températures estivales ambiantes avoisinantes et même dépassant $40^{\circ} \mathrm{C}$ dans les régions envahies de la Tunisie Centrale, caractérisée par ses milieux naturels semi-arides. Les résultats de cette étude accomplie au laboratoire ont mis l'accent sur l'impact du traitement thermique sur la destruction de la viabilité des semences de cette plante envahissante et ont approuvé l'étude des possibilités de destruction des semences par compostage, mode de traitement biologique caractérisé généralement par des températures pouvant atteindre 60 à $70^{\circ} \mathrm{C}$, voire $80^{\circ} \mathrm{C}$.

Cette hypothèse a été confirmée par M'Sadak et al. [19] qui ont rapporté que les baies et les graines de morelle jaune ont été introduites dans trois types de compost (Co-compost sylvicole, Co-compost ovin et compost ovin) à mi-profondeur de chaque tas, en vue d'étudier l'incidence de la température de chaque compost sur la viabilité des graines de SOEL. L'effet thermique a été étudié en établissant les courbes isothermes temporelles à divers endroits et à différentes profondeurs au niveau d'un tas de Co-compost sylvicole [20].

Les résultats de l'essai de germination des graines et des graines issues de baies traitées par compostage n'ont donné aucune plantule de morelle jaune, alors que la faculté germinative des graines témoin (non traitées par compostage) était avoisinante de 60\% [19].

\section{Caractérisation cellulosique de la biomasse partielle de SOEL}

Mustin [17] a classé les composés carbonés en facilement biodégradables (Sucres et Hémicelluloses) et en difficilement biodégradables (Cellulose et Lignine).

Les fortes teneurs en carbone organique relevées au niveau des différentes parties de SOEL, particulièrement au niveau des racines et surtout des tiges, pour les deux stades de croissance étudiés ont incité à la détermination de la teneur en cellulose brute dans les différentes parties de la plante, surtout que ce polymère est indispensable à la nutrition des champignons et des 
bactéries pendant les phases mésophile et thermophile de compostage à l'opposé de la lignine qui prouve son utilité dans des phases plus avancées du compostage [16]. D'après la figure 1 ci-après, on observe des taux trop élevés de cellulose brute au niveau des racines et notamment des tiges de la plante pour les deux stades de croissance étudiés.

L'augmentation du taux de cellulose observé au stade grenaison avancé est d'environ 3,7\% pour les racines et de $2 \%$ pour les tiges, ce qui est dû à l'achèvement de la formation des tissus de soutien propres à ces deux organes de la plante. Néanmoins, l'augmentation assez importante $(9,3 \%)$ de cette teneur observée au niveau de la troisième partie étudiée de la plante (Feuilles + baies) pourrait être expliquée par la formation des fruits à ce stade de croissance (graines enveloppées par une paroi assez rigide ou baie).

Les fortes teneurs en cellulose brute identifiées au niveau des différentes parties de la plante ont confirmé la difficulté de compostage de la biomasse partielle de morelle jaune surtout les tiges et les racines. En contre partie, cette propriété cellulosique de la plante a incité à étudier les possibilités de valoriser la biomasse de SOEL dans le secteur de dépollution des eaux usées, en particulier dans le traitement des effluents textiles par biosorption sur biomasses de cette plante. Pour cette raison, une étude préliminaire concernant les possibilités d'utiliser la biomasse de cette plante envahissante (après broyage) dans le traitement des eaux industrielles a été réalisée et dont les résultats trouvés ont dévoilé des taux de rétention importants d'un colorant textile (bleu de méthylène) par les trois parties testées de SOEL [21].

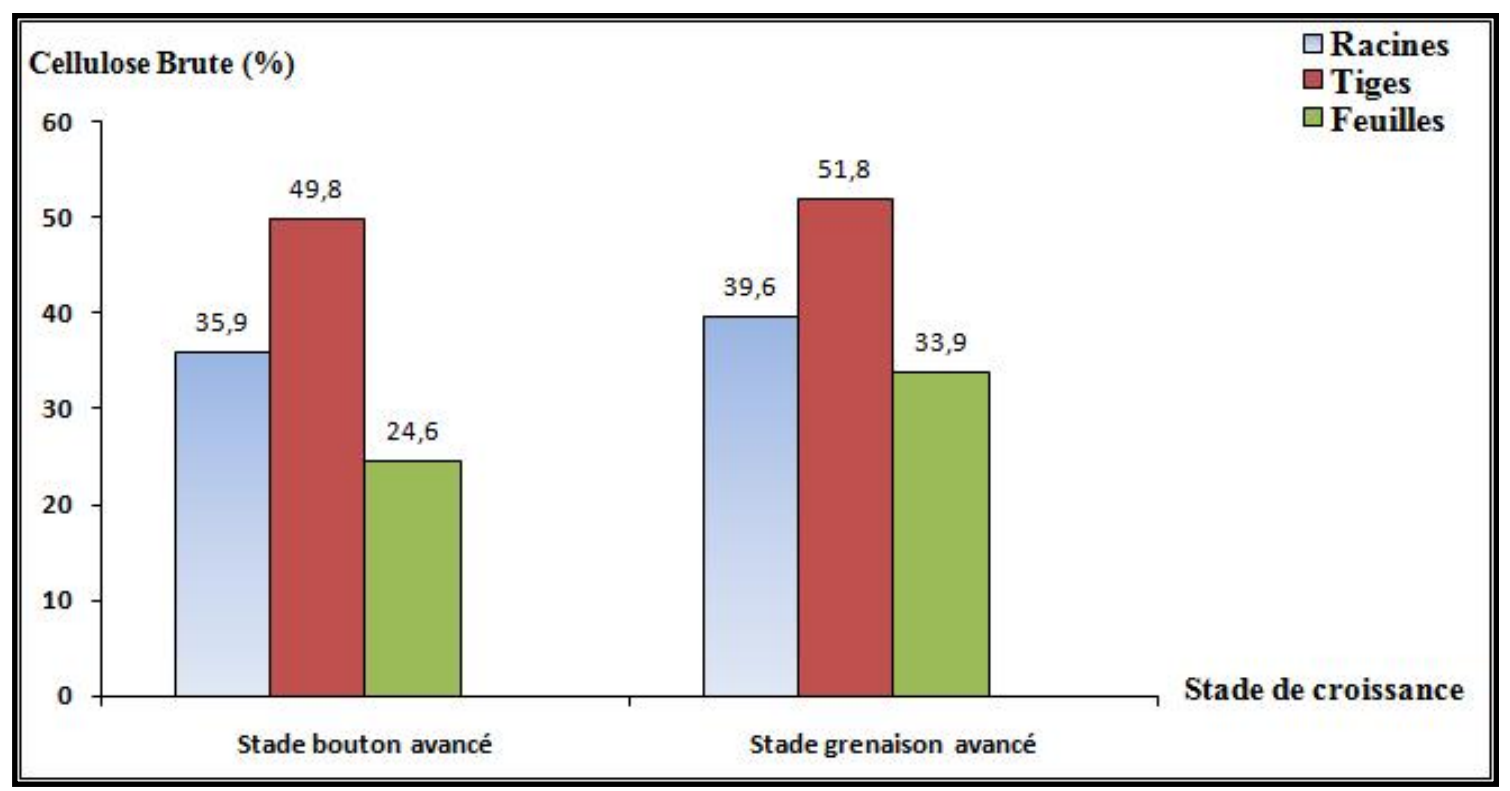

Fig.1. Teneurs en cellulose brute selon le type de biomasse et selon le stade de croissance

\section{Synthèse des valorisations possibles de SOEL}


Cette étude s'est intéressée à la caractérisation qualitative de la biomasse partielle de SOEL, plante envahissante très redoutable en région méditerranéenne, en vue de trouver des possibilités de valorisation des déchets de cette plante sans avoir recours à leur incinération après son arrachage à partir de terres envahies.

Deux voies de valorisation (compostage et dépollution) des déchets de SOEL se sont avérées possibles, toutefois, elles sont fortement dépendantes du stade de croissance de la plante au moment d'arrachage.

* Pour des plantes de morelle jaune arrachées au stade végétatif, en particulier, bouton floral, on recommande le compostage de la plante entière. Les tiges et les racines, déchets secs et ligneux, vont assurer l'apport d'énergie et d'oxygène (agent structurant) aux microorganismes décomposeurs de la matière organique. Les feuilles et les boutons floraux, déchets humides et riches en azote, vont assurer la fermentation rapide et sans aucun arrosage préalable.

* Pour des plantes de morelle jaune arrachées au stade fructifère, en particulier, stade graines mures), deux modes de valorisation sont envisageables. Le premier, étant le Co-compostage ou compostage conjoint de la biomasse totale ou partielle de la plante avec un autre déchet de nature fermentescible (comme le fumier ovin, les tontes de gazon fraîches, les fientes avicoles, ...). Le deuxième, étant le stockage des déchets de la plante (avec prise en considération des risques de dissémination de la plante par graine) en vue d'être ajoutée dans d'autres andains de compost (manquant d'agents structurants) ou même pour être Co-compostée avec les déchets de la même plante de nouveau réapparus (stade croissance végétative).

* La dépollution des eaux résiduaires textiles sur fibres de biomasse partielle ou totale de morelle jaune s'est avérée faisable au stade grenaison avancée, surtout que même les graines ont présenté des capacités biosorbantes de colorants textiles intéressantes.

* Le compostage ou Co-compostage des déchets de morelle jaune chargées en colorants textiles (après utilisation comme support de dépollution) semble aussi être envisageable. Toutefois, il convient d'étudier la cinétique de désorption des colorants par les fibres de SOEL pour s'assurer que l'adsorption des colorants n'est pas réversible ou à la limite partiellement réversible (possibilité d'arrosage de l'andain par l'extrait chargé en colorants désorbés par les fibres de SOEL). 


\section{CONCLUSION}

A la lumière des travaux réalisés relatifs à la caractérisation qualitative de la biomasse produite de SOEL, les enseignements essentiels ci-après peuvent être tirés.

* La détermination des paramètres physico-chimiques (Matière sèche, Humidité, $\mathrm{C} / \mathrm{N}, \mathrm{pH}$, $\mathrm{CE}, \ldots$ ) des différentes parties (racines, tiges et frondaison) et pour deux stades de croissance de la plante (Stade bouton floral avancé et stade grenaison avancé) a été élaborée en vue d'étudier son aptitude au compostage. La richesse en matière carbonée et l'aspect assez sec de la biomasse de cette plante, constatés au niveau des différentes parties et pour deux stades de croissance, ont favorisé la possibilité de son usage en Co-compostage avec d'autres déchets humides et fermentescibles (riches en azote) pour parvenir à une telle valorisation.

* La caractérisation cellulosique des mêmes parties de la plante et pour les mêmes stades de croissance sus-indiqués a mis au point la richesse de cette dernière en cellulose brute au niveau des différents organes étudiés, avec une augmentation de la teneur en cellulose enregistrée au niveau du stade grenaison avancé, due à l'établissement des organes de soutien propres à la plante. La cellulose présente pourrait favoriser le pouvoir de rétention des colorants textiles par les fibres de SOEL.

\section{RÉFÉRENCES BIBLIOGRAPHIQUES}

[1] Mekki M., M'Hafdhi M., Belhaj R., Alrouechdi K. Expérience Tunisienne des Champs. Ecole Paysanne sur la lutte intégrée contre une plante exotique envahissante : Solanum elaeagnifolium. Proceedings of the 2nd International Workshop on Invasive Alien Plants in the Mediterranean Type Regions of the World, Trabzon, Turkey, August 2-6 2010, 240-248. [2] AME, ARPE-PACA. Plantes envahissantes de la région méditerranéenne. Solanum elaeagnifolium (Cav.) - Agence méditerranéenne de l'environnement, Agence Régionale pour l'Environnement Provence-Alpes-Côte d'Azur, 2003, 2 p. www.invmed.fr/sites/invmed.fr/files/ficheAMEsolanum.pdf

[3] Marco A., Leblay E. Fiche Espèce Solanum elaeagnifolium Cavanilles, Fédération des Conservatoires Botaniques Nationaux, 2010, 4 p.

http://www.centrederessources-

loirenature.com/mediatheque/especes_inva/fiches_FCBN/Fiche\%20-

\%20solanum\%20elaeagnifolium_sr.pdf 
[4] Chalghaf E., Aissa M., Mellassi H., Mekki M. Maîtrise de la propagation de la morelle jaune (Solanum elaeagnifolium Cav.) dans le gouvernorat de Kairouan (Tunisie)- Bulletin EPPO/OEPP, 2007, 37 (1), 132-136.

[http://www.ingentaconnect.com/content/bsc/eppo/2007/00000037/00000001/art00030]

[5] Mekki M. Biology, distribution and impacts of silverleaf nightshade (Solanum elaeagnifolium Cav.). EPPO Bulletin, 2007, 37 (1), 114-118.

[6] Mekki M. Potential threat of solanum elaeagnifolium Cav. to the Tunisian fields. Invasive Plants in Mediterranean Type regions of the world, 2006, 165-170. Environmental Encounters Series ${ }^{\circ} 59$, Council of Europe Publishing. Atelier international sur les plantes envahissantes des régions de type méditerranéen, 25-27, Mai 2005, Mèze (Hérault), France.

[7] Bouhache M., Ameur A. Synthèse des Travaux effectués au Maroc. Projet de Morelle Jaune (Solanum elaeagnifolium Cav.). Ministère de l'Agriculture et de la Mise en Valeur Agricole, Maroc, 1994, 17-124.

[8] Mekki M. Distinction between weed control and invasive alien plant management approaches: case study of Solanum elaeagnifolium management in North african countries. Proceedings of the International symposium on system intensification towards food and environmental security, organized by the Crop and Weed Science Society and Bidhan Chandra Krishi Viswavidyalaya, February 24-27 2011, 16-18.

[9] Organisation Euro-méditerranéenne de Protection des Plantes (OEPP). Fiches informatives sur les organismes de quarantaine - Solanum elaeagnifolium. Bulletin OEPP/EPPO, $\mathrm{N}^{\circ}$ 37, 2007, 236-245. [http://www.eppo.org/QUARANTINE/plants/ Solanum_elaegnifolium/Solanum_elaeagnifolium_DS.pdf]

[10] AFNOR. Aliments des animaux. Détermination de la teneur en eau, NF-18-109, Octobre $1982,5 \mathrm{p}$.

[11] AOAC (Association of Official Analytical Chemist). Official Methods of Analysis, 16 th ed. AOAC, Washington, DC, 1990.

[12] Golueke C.G. Golueke, Biological processing: composting and hydrolysis. In: D.G. Wilson, Editor, Handbook of Solid Waste Management, Van Norstrand Reinhold, New York, 1977, 197-225.

[13] Gerbaud E., T. Dutoit, A. Barroit, B. Toussaint. Teneurs en minéraux de fourrages de chaumes et de leurs adventices : l'exemple d'une exploitation agricole du Sud-Est de la France (Vaucluse). Anim. Res., 2001, 50, 495-505. 
[14] Trimech I. Faisabilité de l'éradication de la morelle jaune dans le périmètre public irrigué de Chott Mariem (Sousse), Stage de fin d'études, I.S.A. Chott-Mariem, Tunisie, 2008, 50 p. [15] Anger D. La normalisation : Principe et fonctionnement, Norme AFNOR NF U 44-051, 2005. [www.reseau-ideal.asso.fr/medias/it/sytrad\%202006/sp2/d_anger.pdf]

[16] Goeden R.D. Insect ecology of silverleaf nightshade. Weed Science, 1971, 19, 45-51.

[17] Mustin M. Le compost : Gestion de la matière organique. Ed. François Dubusc, Paris, 1987, 954 p.

[18] M'Sadak, Y., Saad, I., Saidi, D. Évaluation de la capacité germinative des semences de Morelle Jaune après traitement thermique, Communication par Affiche, $16^{\text {ème }}$ Journées Scientifiques IRESA, 2-3 Décembre 2009, Nabeul, Tunisie.

[19] M'Sadak Y., Saad I., Saidi D. Contrôle de la destruction de la viabilité des semences de morelle jaune par le compostage. Communication par Affiche, Association Tunisienne des Sciences Biologiques (ATSB), $21^{\text {ème }}$ Forum, 15-18 Mars 2010, Mahdia, Tunisie.

[20] M'Sadak Y., Saad I., Saidi D. Suivi et Analyse thermiques du processus de Co-compostage sylvicole dans une pépinière forestière moderne (Tunisie), Journal des Sciences Fondamentales et Appliquées, 2013, 5 (1), 1-12.

[jfas.info/index.php/JFAS/article/download/94/pdf]

[21] Saad I. Caractérisation Qualitative et Valorisation de la Biomasse de la Morelle Jaune par Compostage et comme Biosorbant des Colorants Textiles, Mastère Agriculture durable, I.S.A. Chott Mariem, Tunisie, 2010, 64 p.

\section{How to cite this article:}

M'Sadak Y, Saad I. Characterization qualitative of soel for a valorization best of the biomass produced. J Fundam Appl Sci. 2015, 7(1), 33-48. 